\title{
Study on a Measuring Method of Permittivity Distribution of Functionally Graded Material
}

\author{
Masashi Ohchi ${ }^{1}$, Susumu Konno ${ }^{1}$, Hiroaki Sugaya ${ }^{1}$, Tatsuya Furukawa ${ }^{2}$, \\ Hideaki Itoh $^{2}$ and Hisao Fukumoto ${ }^{2}$ \\ ${ }^{1}$ Department of Electrical, Electronics and Computer Engineering, \\ Faculty of Engineering, Chiba Institute of Technology \\ ${ }^{2}$ Graduate School of Science and Engineering, Saga University \\ masashi@mem.iee.or.jp,skonno@ieee.org
}

\begin{abstract}
To improve the insulation performance of high voltage equipment, new functional materials of distributed permittivity, or the functionally graded materials (FGMs), have been developed. However, methods for measuring the permittivity distribution of these materials have yet to be established. In this study, numerical analyses have been conducted in order to investigate how the position of dielectrics in a cylindrical measurement device affects the capacitance and electric potential within the device. It has been confirmed that the capacitance and electric potential change with a varying position of the dielectrics. Therefore, it is suggested that the permittivity distribution of a given FGM can be estimated by measuring the capacitance and electric potential around it.
\end{abstract}

Keywords: FGM (Functionally Graded Material), Permittivity distribution, Electric field analysis, Capacitance, Electric potential

\section{Introduction}

Developing highly efficient power equipment has become necessary in recent years for building power systems that respond to environmental issues and energy conservation. To increase the efficiency and performance of power equipment, solid insulation techniques will be required.

For this reason, research on new functional materials with graded permittivity to increase insulation capability has been conducted [1]. The new functional materials are integrated materials whose permittivity is not spatially constant. They have the properties that, with appropriate variations in materials constants, the electric field distribution within and surrounding the material can be better controlled than in materials with fixed materials constants. Although they can be produced, they have been feasible for use because techniques for verifying whether permittivity is actually graded have not been established yet. When a reliable measurement method of distributed permittivity is established, it is expected to have applications in a wide variety of engineering fields.

In the past research, the capacitance of a target object having non-uniform permittivity is measured from its periphery, and the permittivity distribution inside the object is estimated by extrapolation [2-10]. In those studies, not only measurement experiments have been conducted, but a numerical analysis of an electric field using the finite element method has been reported. The numerical analysis conducted in the past, however, was a that of capacitance alone, using relatively coarse mesh data in a two-dimensional model [9]. Thus, in 
this study, in order to improve the estimation precision of distributed permittivity, the numerical analysis has been conducted using a three-dimensional model while focusing on electric potential in space between two additional electrodes. Numerical analysis has been carried out by modeling a device with one or two objects (acrylic rods) in three dimensions. In this three-dimensional modeling, the angle of rotation of object has been changed from 1degree steps to 6-degree steps due to the issue of computation time.

We propose a cylindrical device for measuring the permittivity distribution of a given object. The target object to be measured is rotated in the central hollow of the device. To investigate fundamental measuring capabilities of the device, we carry out numerical analyses of the cases where the target object consists of one or two acrylic rods. As a result, it is confirmed that different values of the capacitance and electric potential are obtained depending on the positions of the acrylic rods. This result suggests that there is a possibility that the permittivity distribution can be estimated by our device.

\section{FGM (Functionally Graded Material) and Measurement Method}

\subsection{FGM (Functionally Graded Material)}

A schematic view of the functional material with graded permittivity is shown in Figure 1. Figure 1(a) shows a uniform material of permittivity, (b) is a compound material. Figure 1(c) shows the FGM (Functionally Graded Material), whose permittivity changes continuously in the $x$ direction. To simulate this functional material with graded permittivity, we use one or two dielectric rods. An example in which the FGM is modeled by two rods is shown in Figure 2(a). The rods are rotated inside the experimental device as shown in Figure 2(b) and (c), and capacitance and electric potential are measured.

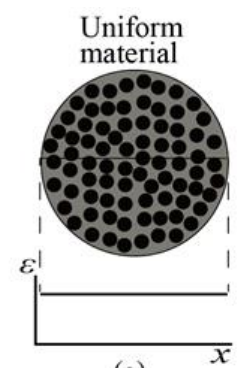

(a)

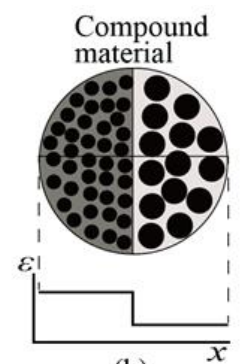

(b)

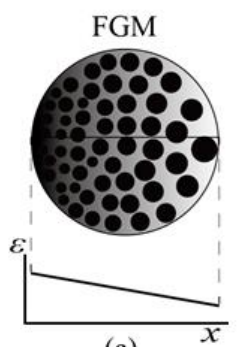

(c)

Figure 1. Functionally graded material(FGM)

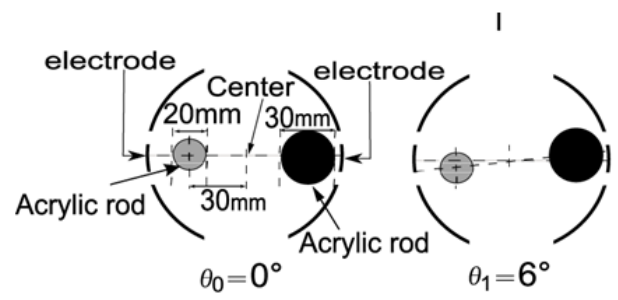

(a)

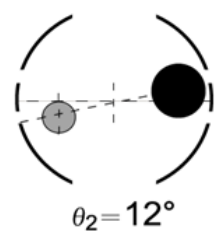

(c)

Figure 2. Simulated FGM and evaluation method with the rotating acrylic rod 


\subsection{Evaluation method}

Figure 3 shows the photo of the experimental measurement device proposed by the authors. The experimental device is constructed from two acrylic pipes (height $300 \mathrm{~mm}$, thickness $10 \mathrm{~mm}$, outside diameter of inner pipe $96 \mathrm{~mm}$, outside diameter of outer pipe $212 \mathrm{~mm}$ ). Measurement electrodes and additional electrodes are put on the inside of the inner acrylic pipe. Shields are mounted on the outsides of both acrylic pipes.

Figure 4 illustrates an equivalent circuit of the evaluation device. By the additional electrodes, it becomes possible to measure only the current that flows through the measurement electrodes. Stray capacitance and edge effects are reduced even at long intervals, and precise measurement becomes possible. Acrylic rods (height $300 \mathrm{~mm}$, diameter $20 \mathrm{~mm}, 25 \mathrm{~mm}$ and $30 \mathrm{~mm}$ ) are introduced as the simulated FGMs. As shown in Figure 4, the left side is called the high-potential measurement electrode to which a voltage source is connected, and the right side is called the low-potential measurement electrode to which an ammeter is connected. The measurement electrode area is $18 \mathrm{~mm} \times 18 \mathrm{~mm}$, and the distance between the measurement electrodes is $96 \mathrm{~mm}$. The additional electrodes surround the measurement electrodes in a 120-degree arc on both the high-potential side and low-potential side, and each gap between the measurement electrodes and additional electrodes is $2 \mathrm{~mm}$.

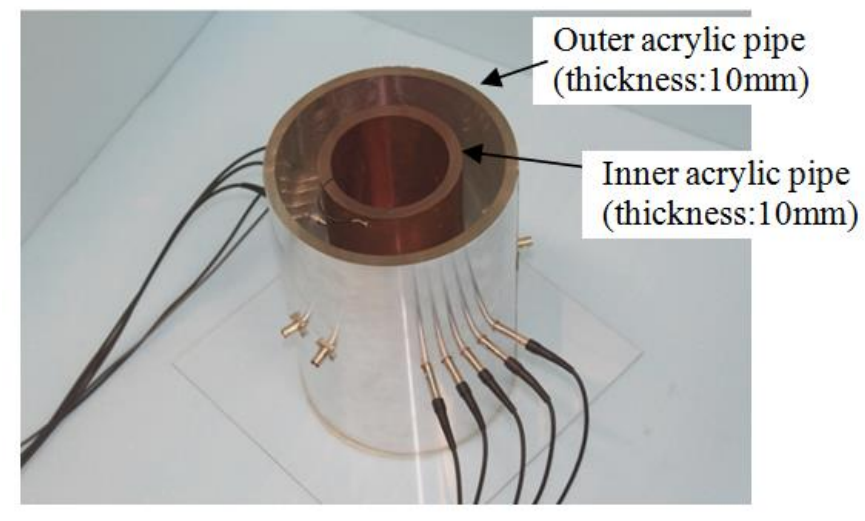

\section{Figure 3. Photo of the experimental device}

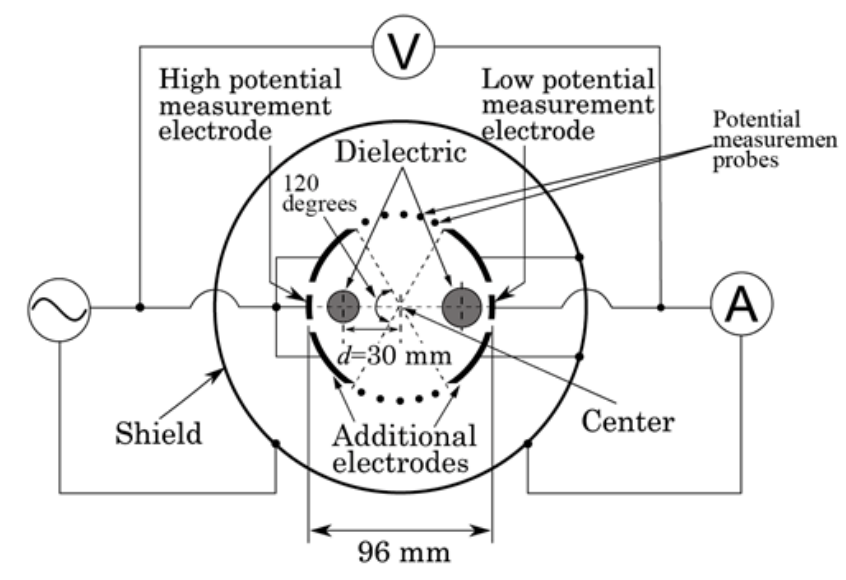

Figure 4. Equivalent circuit of evaluation method with measuring capacitance and electric potentials 


\section{Analytical modeling of the experimental device}

\subsection{Numerical Analysis Model}

An overall view of the analysis model of the experimental device is shown in Figure 5. A cross-sectional diagram is shown in Figure 6. Then, electric potential was computed at positions Ch1 through Ch10 in Figure 6, and capacitance was computed on the low-potential measurement electrode by the following technique.

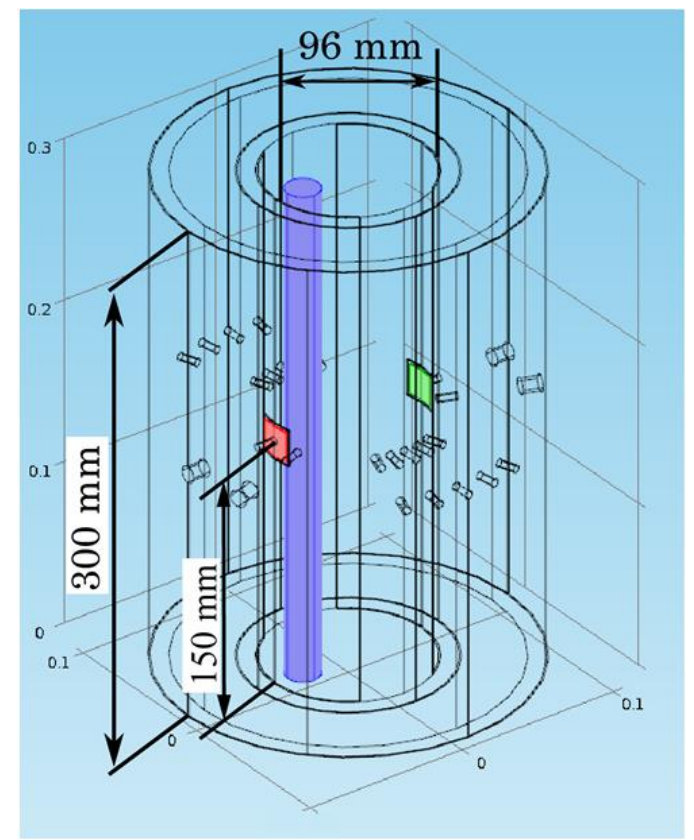

Figure 5. Overall view of the model for analysis

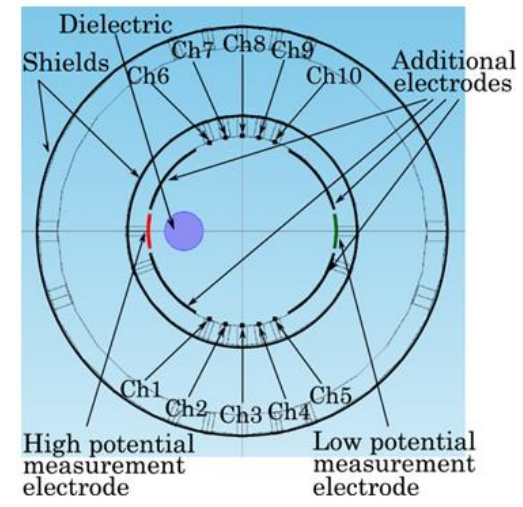

Figure 6. Cross-sectional view of the model for analysis

\subsection{Analysis Technique}

The acrylic rods were placed in the experimental device, and numerical electric field analysis was executed using the finite element method by rotating the rods 360 degrees counterclockwise, 6 degrees every step, from the high-potential measurement electrode. The computation time at each angle was approximately 20 minutes. As interface conditions, the 
high-potential measurement electrode was set at $2 \mathrm{~V}$, the low-potential measurement electrode was set at $0 \mathrm{~V}$, and the shields were grounded. The materials and relative permittivities of the analysis model are given in Table 1 .

Table 1. Materials of model for analysis

\begin{tabular}{|l|l|l|}
\hline Medium & Domain & $\begin{array}{l}\text { Relative } \\
\text { permittivity }\end{array}$ \\
\hline Air & Air & 1.0 \\
\hline Copper & $\begin{array}{l}\text { Electrode plate } \\
\text { Shield of the } \\
\text { inner frame }\end{array}$ & 1.0 \\
\hline Aluminum & $\begin{array}{l}\text { Shield of outer } \\
\text { frame }\end{array}$ & 1.0 \\
\hline $\begin{array}{l}\text { Acrylic } \\
\text { plastic }\end{array}$ & $\begin{array}{l}\text { Acrylic pipe } \\
\text { Acrylic rod }\end{array}$ & $\begin{array}{l}3.05 \\
3.05\end{array}$ \\
\hline
\end{tabular}

\subsection{Capacitance computation method}

The technique for computing capacitance by numerical computation was as follows. First, charge $Q$ was computed by equation (1). $\boldsymbol{D}=\left(D_{x}, D_{y}, D_{z}\right)$ is the electric flux density on the low-potential measurement electrode, $\boldsymbol{n}$ is the unit normal vector on the low-potential measurement electrode, and $S$ is the integration region (which is the surface of the lowpotential measurement electrode).

$$
Q=\int_{S} \mathbf{D} \bullet \mathbf{n} d s \ldots \ldots \cdot \ldots \cdot \ldots \cdot \ldots \cdot \ldots
$$

Then, the result of formula (1) was divided by the potential difference $V$ between the highpotential and low-potential electrodes to compute capacitance $C$ as shown in equation (2).

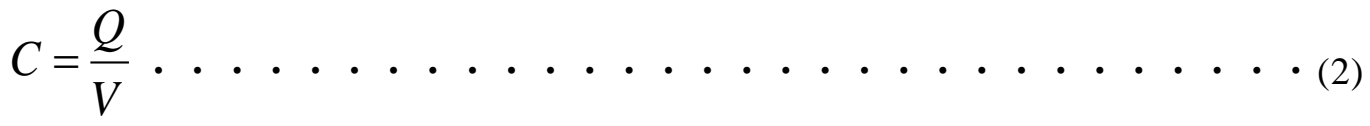

\section{Numerical analysis results}

4.1. Comparison of analysis results and experimental results regarding the capacitance of an acrylic rod of diameter $20 \mathrm{~mm}$ at position $30 \mathrm{~mm}$ away from the center of the experimental device

The results of comparing analysis results and experimental results and regarding the capacitance of an acrylic rod of diameter $20 \mathrm{~mm}$ at a dielectric position of $30 \mathrm{~mm}$ away from the center of the experimental device are shown in Figure 7. From this figure it was confirmed that the maximum value near 180 degrees and the minimum value near 240 degrees were similar in the analysis results and experimental results. It was further confirmed that the overall trend of changes was also similar. However, the analysis results from 6 degrees to 132 degrees differed slightly from the experimental results. However, since the maximum value and trend of changes were similar, it is considered that the numerical analysis results are reasonable. 
In case of the maximum and minimum capacitance, the electric flux lines are shown in Figures 8 and 9, respectively. The rotation angle at the maximum value was 180 degrees, and the rotation angle at the minimum value was 234 degrees.

It was seen that the dielectric flux distribution entering the low-potential measurement electrode changes depending on the dielectric rotating angle. It was confirmed that the dielectric flux entering the low-potential measurement electrode is more dense in Figure 8, where capacitance is high, than in Figure 9.

Thus, the changes in displacement current were confirmed by drawing the electric flux distribution.
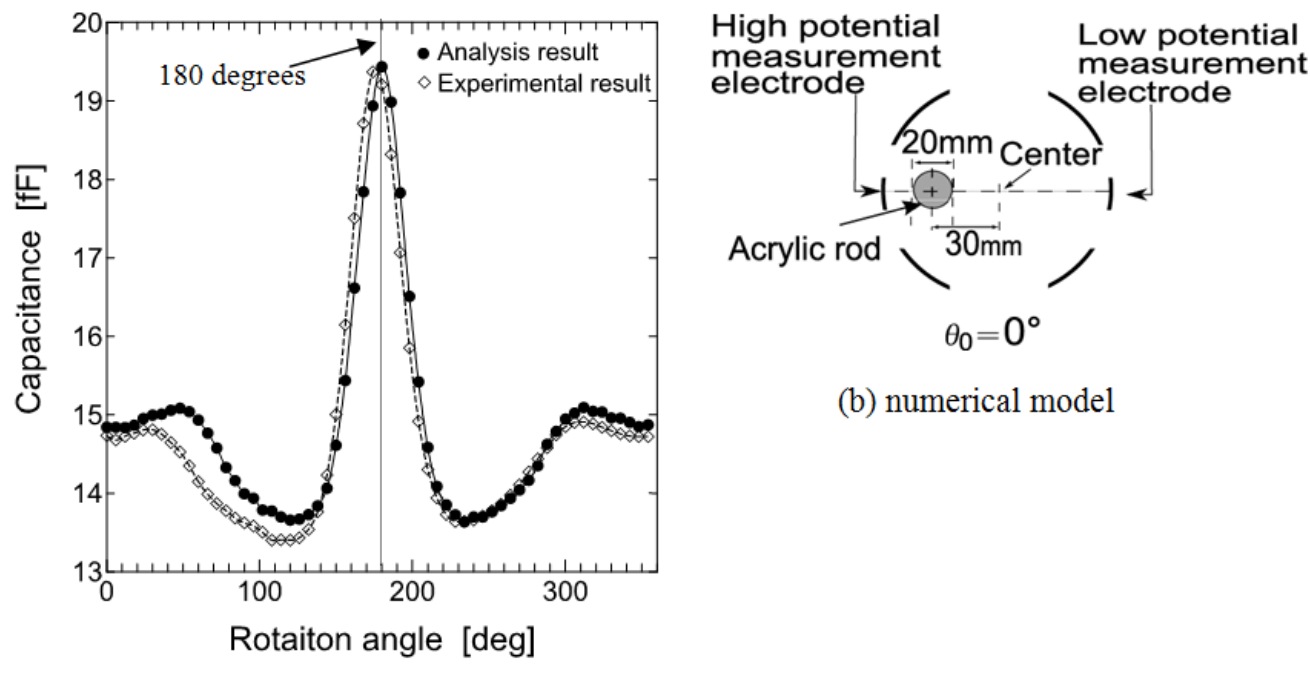

(a) results of analysis and experiment

(b) numerical model

Figure 7. Comparison between analysis results and experimental results for an acrylic diameter of $20 \mathrm{~mm}$ at position $30 \mathrm{~mm}$ away from the center of the experimental device

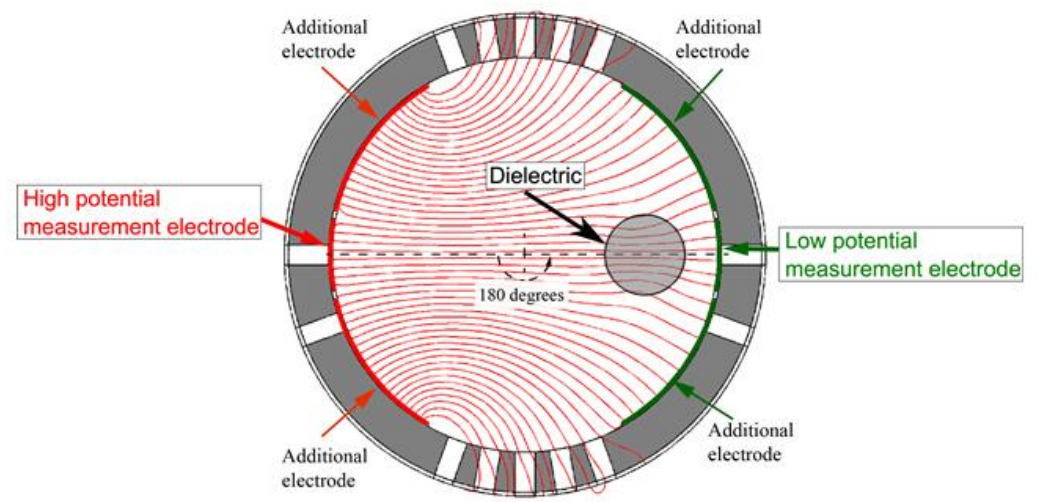

Figure 8. Electric flux lines for the case of maximum capacitance (acrylic diameter, $20 \mathrm{~mm}$; rotation angle, 180 degrees) 


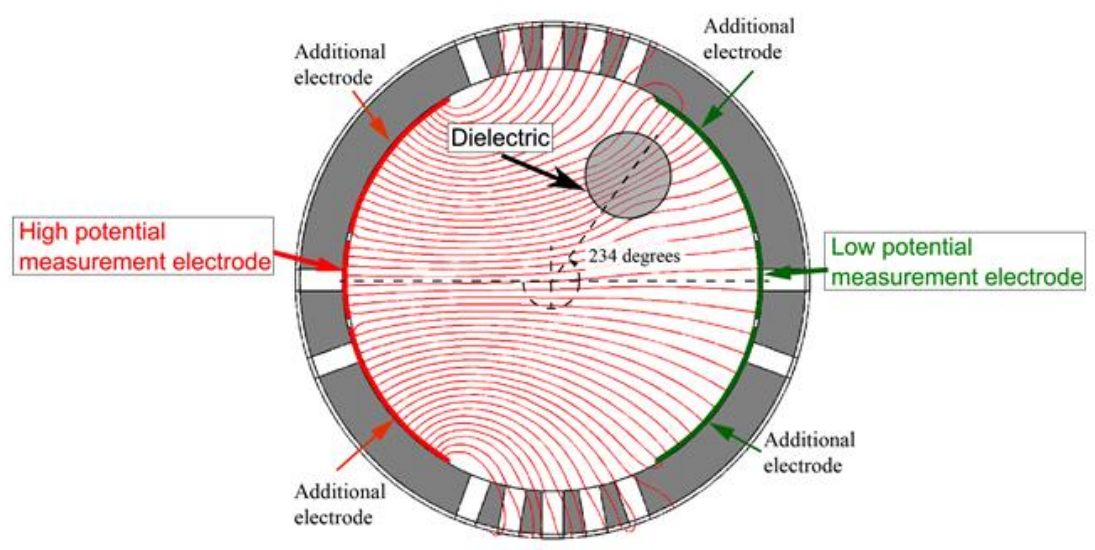

Figure 9. Electric flux lines for the case of minimum capacitance (acrylic diameter, $20 \mathrm{~mm}$; rotation angle, 234 degrees)

\subsection{Results of two acrylic rods of diameter $20 \mathrm{~mm}$ at position $30 \mathrm{~mm}$ away from the center of the experimental device}

A cross-sectional view of the model for analysis at rotation angle 0 degrees is shown in Figure 10. Furthermore, the capacitance results of two acrylic rods of diameter $20 \mathrm{~mm}$ at a position of $30 \mathrm{~mm}$ away from the center are shown in Figure 11. Electric potential results of each channel are shown in Figure 12. It was confirmed that capacitance and electric potential also change depending on dielectric position when there are two acrylic rods.

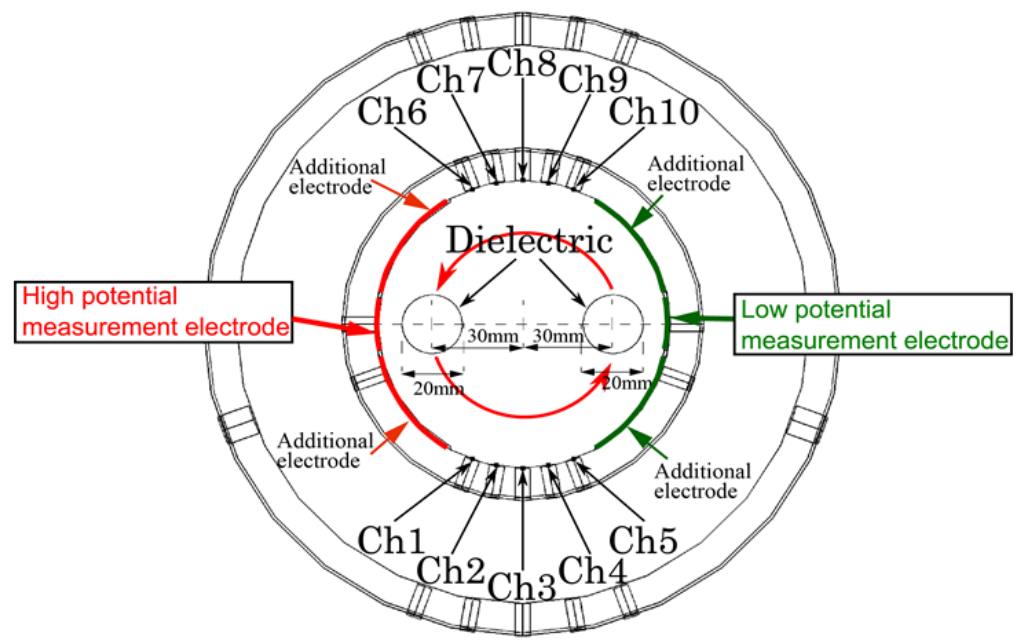

Figure 10. Cross-sectional diagram of two acrylic rods with identical diameters at a rotation angle of 0 degrees 


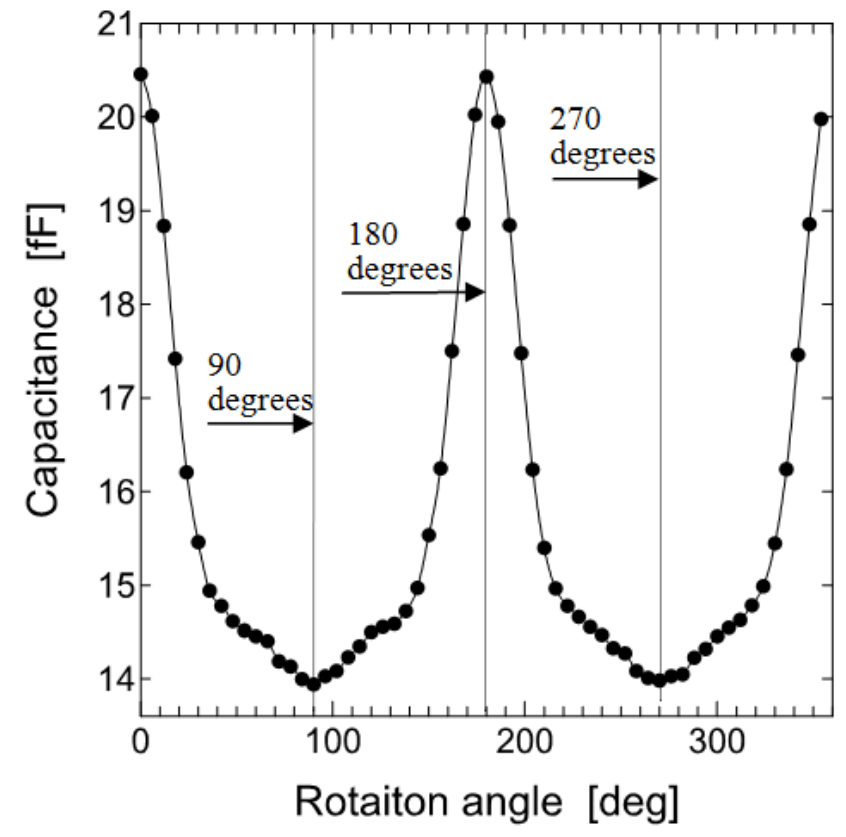

Figure 11. Numerical analysis of capacitance for the case of two dielectric rods with identical diameters

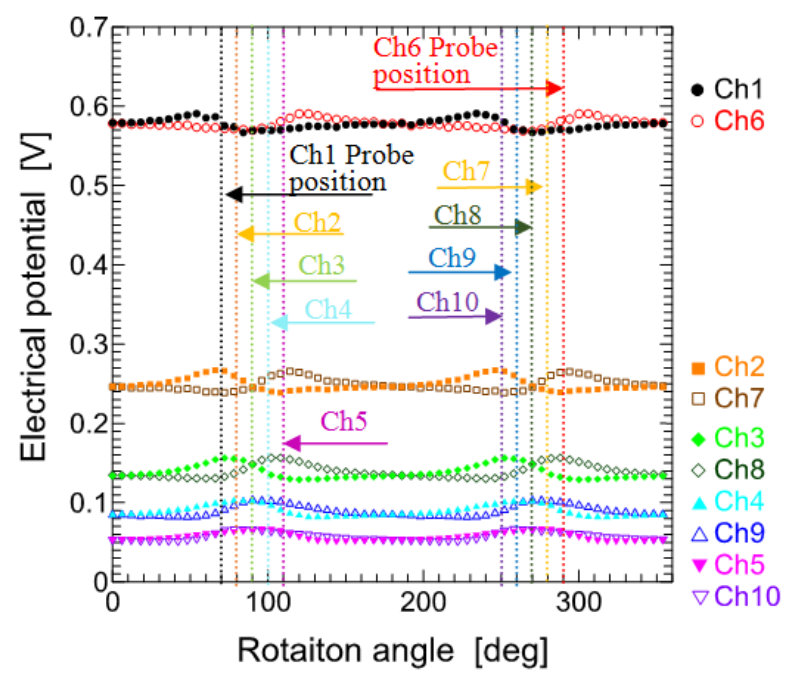

Figure 12. Numerical analysis of electric potential for the case of two dielectric rods with identical diameters

It was seen that capacitance reached its maximum values at the rotation angles of 0 and 180 degrees, where one of the acrylic rods was in front of the low-potential measurement electrode. It was also seen that it reached its minimum values near 90 and 270 degrees. The maximum and minimum values are higher than those when there is only one dielectric. In particular, it was shown that the maximum value was about $1 \mathrm{fF}$ higher. It was also seen that electric potential changes when an acrylic rod is located in the vicinity of the measurement location (each channel's position is shown in Figure 10). 


\subsection{Results of acrylic rods of diameter $20 \mathrm{~mm}$ and $30 \mathrm{~mm}$ at position $30 \mathrm{~mm}$ away from the center of the experimental device}

A cross-sectional view of the model for analysis at rotation angle 0 degrees is shown in Figure 13. Capacitance results of acrylic rods of diameter $20 \mathrm{~mm}$ and $30 \mathrm{~mm}$ at a dielectric position $30 \mathrm{~mm}$ away from the center is shown in Figure 14.

The electric potential at each channel is shown in Figure 15. It was confirmed that capacitance and electric potential also change depending on the position of the two dielectrics. It was seen that capacitance reaches its maximum value at a rotation angle of 0 degrees, where the acrylic rod of diameter $30 \mathrm{~mm}$ is in front of the low-potential measurement electrode.

It was also seen that it reaches its minimum values near 80 and 280 degrees. It was proven that the maximum and minimum values are higher than when there is only one dielectric. In particular, large changes were seen when the acrylic rods of diameter $20 \mathrm{~mm}$ and $30 \mathrm{~mm}$ were in front of the low-potential measurement electrode.

It was also evident that electric potential changes when an acrylic rod is located in the vicinity of the measurement location. It was confirmed that the maximum and minimum for each channel were reached when the rod of diameter $30 \mathrm{~mm}$ was in the vicinity of the measurement location.

\subsection{Results of two rods with different diameters}

The capacitance results of two acrylic rods (diameters 20 and $20 \mathrm{~mm}, 20$ and $25 \mathrm{~mm}$, and 20 and $30 \mathrm{~mm}$ ) at a dielectric position of $30 \mathrm{~mm}$ away from the center are shown in Figure 16. For the reason, the case of one dielectric substance is introduced. Capacitance results of three models at the rotation angle of 180 degrees change depending on the diameters of the dielectrics. The electric potential at channel 3 is shown in Figure 17. It was confirmed that capacitance and electric potential also change depending on the position of the dielectrics when there are two cylindrical acrylic rods in the experimental device. The capacitance value shows the local maximum in the case of the dielectric position being nearest to the low potential measurement electrode (that is, when rotation angle is 180 degrees). However, from Figure 17, the electric potential reaches its local maximum values at a rotation angle of 72 or 78 degrees and 252 or 258 degrees. Those angles are shifted from the position of channel 3 by approximately 18 or 12 degrees toward high-potential measurement electrode side. In addition, the local minimum values of the electric potential is shifted from position of channel 3 by approximately 30 or 36 degrees to the low-potential measurement electrode side. 


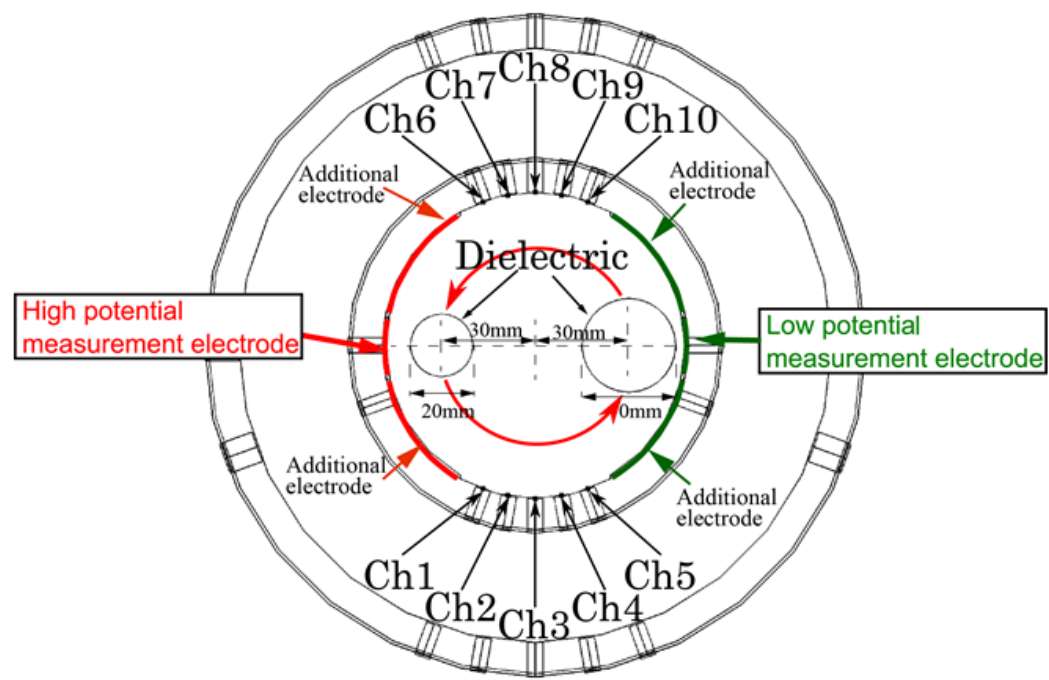

Figure 13. Cross-sectional diagram of two acrylic rods with different diameters at a rotational angle of 0 degrees

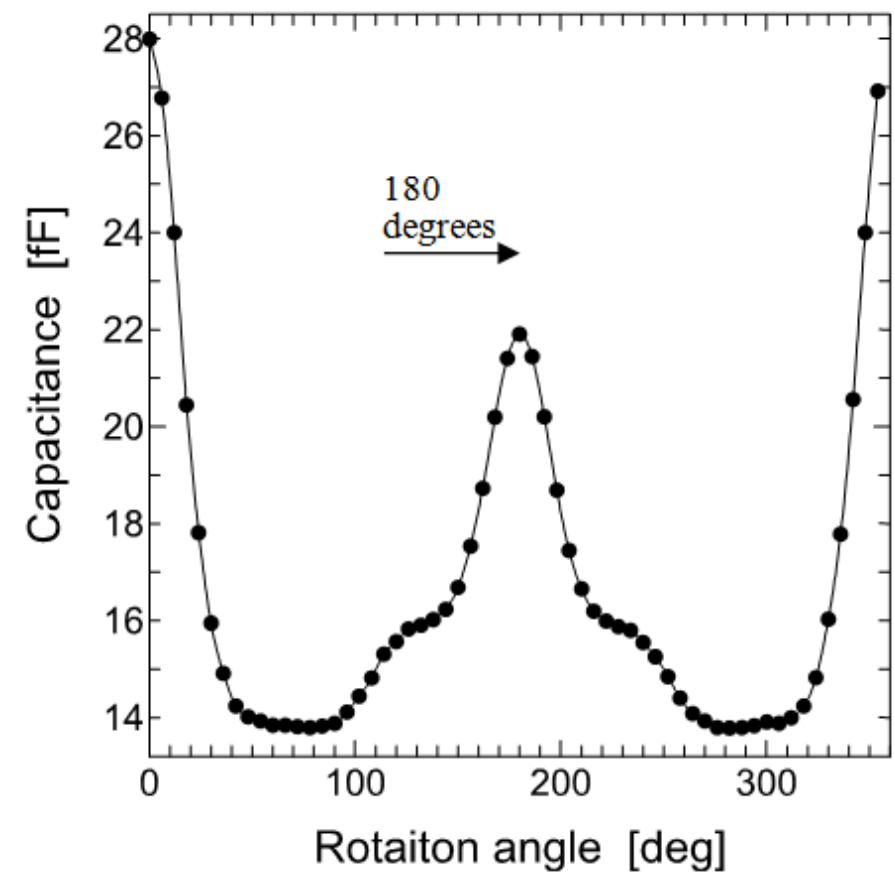

Figure 14. Numerical analysis of capacitance for the case of two dielectric rods with different diameters 


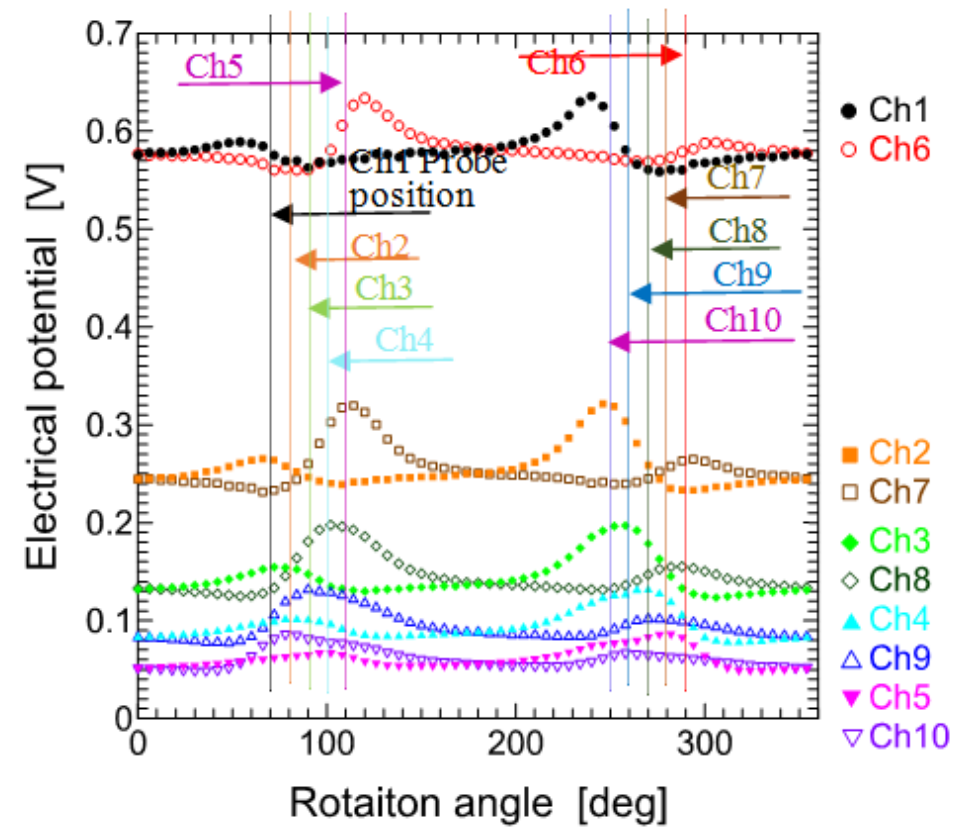

Figure 15. Numerical analysis of electric potential for the case of two dielectric rods with different diameters

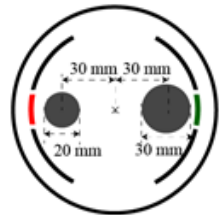

(a) Diameter $20 \mathrm{~mm}, 30 \mathrm{~mm}$

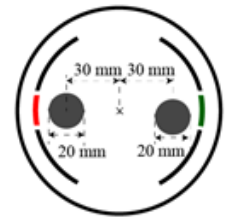

(c) Diameter $20 \mathrm{~mm}, 20 \mathrm{~mm}$

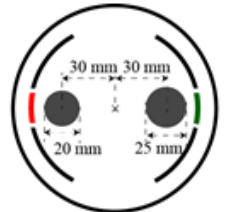

(b) Diameter $20 \mathrm{~mm}, 25 \mathrm{~mm}$

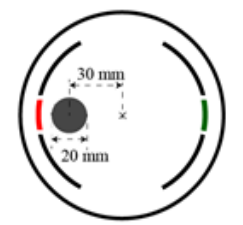

(d) Diameter $20 \mathrm{~mm}$

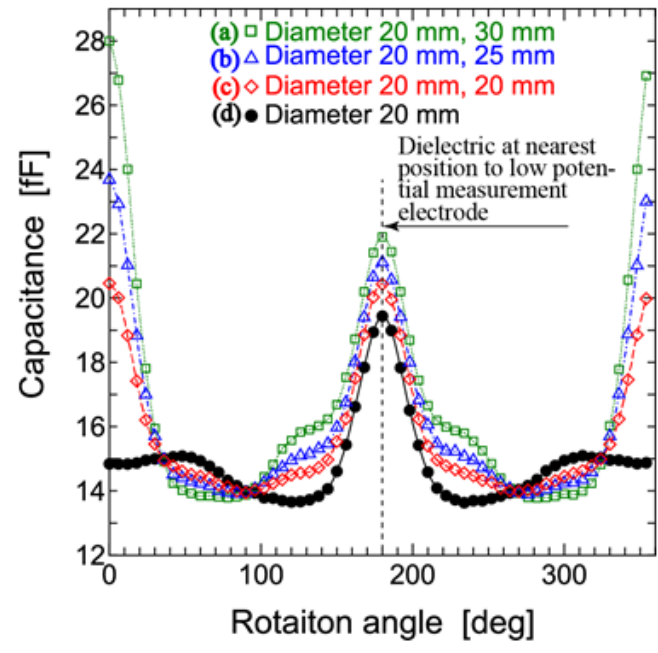

Figure 16. Numerical analysis of capacitance, comparing two dielectric rods with different diameters and a rod 


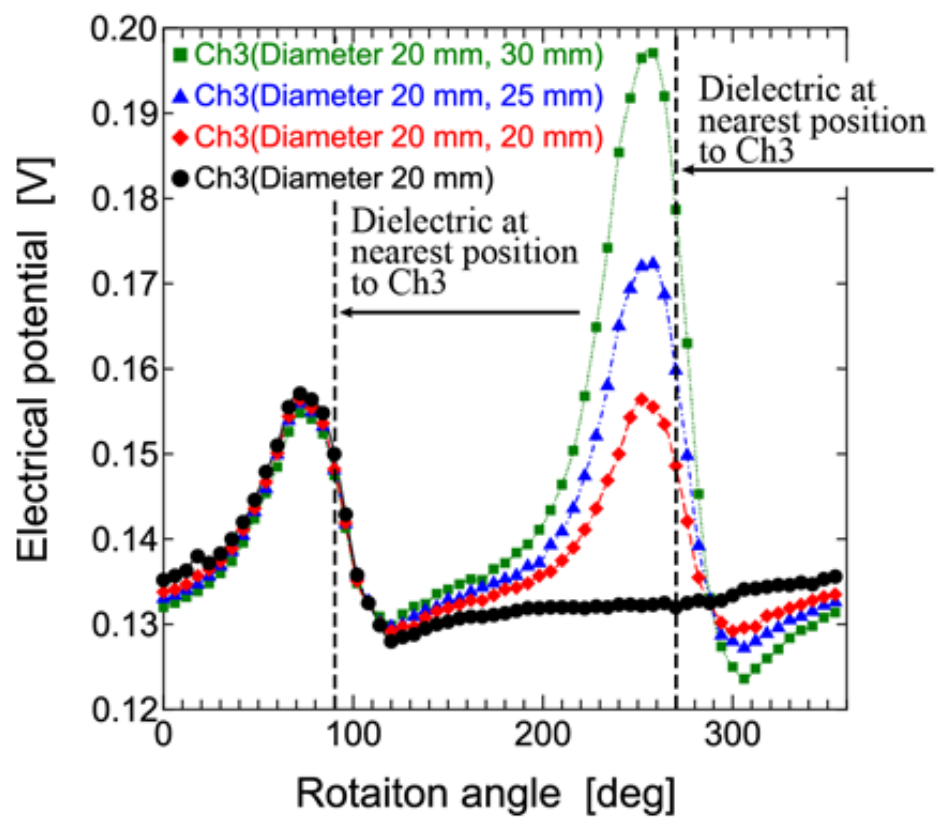

Figure 17. Numerical analysis of electric potential at channel 3, comparing two dielectric rods with different diameters and a rod

\section{Conclusion}

In this paper, how capacitance and electric potential depend on the positions and sizes of dielectrics in a cylindrical measurement device was studied based on a three-dimensional numerical analysis using the finite element method. The analysis was performed by rotating the dielectrics 360 degrees counterclockwise, 6 degrees every step, from the high-potential measurement electrode.

With regard to capacitance, two maximum values were seen when there were two dielectric rods, and dielectric rods of larger diameter resulted in higher maximum capacitance values. Thus, it was confirmed that the diameter of a dielectric rod can be estimated based on the maximum capacitance value.

As a result, it became clear for the first time that the rotation angles in case of local maximum and minimum values of electric potential were shifted from the measurement position of electric potential.

We thought that if electric potential surrounding the experimental device were measured, it would be possible to estimate the dielectric position, and using capacitance and electric potential values with rotating object, the possibility of reconstructing distributed permittivity was greatly improved.

As future topics, we plan to execute a numerical analysis and carry out experiments for more complex permittivity distributions. 


\section{References}

[1] K. Kato, M. Kurimoto, H. Shumiya, H. Adachi, S. Sakuma and H. Okubo, "Application of Functionally Graded Material for Solid Insulator in Gaseous Insulation System”, IEEE Transactions on Dielectrics and Electrical Insulation, vol. 13, no. 1, (2006) February, pp. 362-372.

[2] M. Ohchi, T. Furukawa and H. Shimada, "Novel Scheme for Drawing Electric Lines of Force Using Scalar Potential", IEEE Transactions on Magnetics, vol. 33, no. 2, (1997) March, pp. 1200-1203.

[3] H. Jain, D. Isaacson, P. M. Edic and J. C. Newell, "Electrical Impedance Tomography of Complex Conductivity Distributions with Noncircular Boundary", IEEE Transactions on Biomedical Engineering, vol. 44, no. 11, (1997) November, pp. 1051-1060.

[4] A. Kimoto, Y. Matsuoka and K. Shida, "Reconstruction of temperature change distribution inside the brain phantom using capacitance measurement method", IEEE EMBS Conference, vol. 20, no. 2, (1998), pp. 10581059.

[5] M. Ohchi and T. Furukawa, "Numerical Electric Field Analysis of Simulated Human Brain", IEEE Transactions on Magnetics, vol. 36, no. 4, (2000) July, pp. 712-716.

[6] Z. Bin, X. Chuanlong, Y. Daoye, W. Simin and W. Xin, "Nonlinear image reconstruction using a GA-ECT technique in electrical capacitance tomography", Flow Measurement and Instrumentation, vol. 18, ELSEVIER, (2007), pp. 285-294.

[7] M. Ohchi, T. Furukawa and H. Tokushima, "Feasibility Study on Estimation of Permittivity Distribution by using Genetic Algorithm and Finite Element Analysis", IEEJ Transactions on Electrical and Electronic Engineering, vol. 3, no. 2, (2008) March, pp. 236-243.

[8] J. Mirkowski, W. T. Smolik, M. Yang, T. Olszewski, R. Szabatin, D. S. Radomski and W. Q. Yang, "A New Forward-Problem Solver Based on a Capacitor-Mesh Model for Electrical Capacitance Tomography”, IEEE Transactions on Instrumentation and Measurement, vol. 57, no. 5, (2008) May, pp. 973-980.

[9] M. Ohchi, T. Furukawa, H. Fukumoto and S. Nagamachi, "Effect of Measured Capacitance on Arc Additional Electrodes using Long Distance Measurement Electrodes for Estimation of Permittivity Distribution”, The Institute of Electrical Engineers of Japan Trans. FM, vol. 129, no. 4, (2009) April, pp. 190196, (In Japanese).

[10] S. Okada, M. Ohchi, T. Furukawa, H. Itoh and H. Fukumoto, "Measurement of Capacitance and Electrical Potential of Plural Points for Estimation of Permittivity Distribution", The Institute of Electrical Engineers of Japan Trans. FM, vol. 133, no. 4, (2013) April, pp. 173-179, (In Japanese).

\section{Authors}

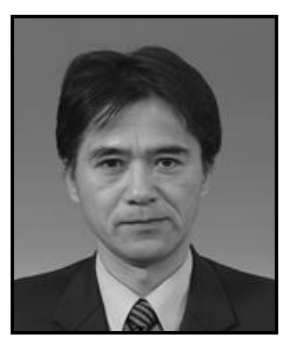

\section{Masashi Ohchi}

He was born in 1964. He received B.E and M.E degrees in 1987 and 1989 respectively from Nagasaki University, Nagasaki, Japan. He joined Nihon Telephone Telegraph Co. in 1989. He was adopted as a research associate at Saga University, Saga, Japan. He received the D.E degree from Kyushu University, Japan in 2000. He was an associate professor of the Department of Electrical and Electronic Engineering at Saga University since 2000. He has moved to Chiba Institute of Technology, Chiba, Japan as a professor in 2008. He is a professor of the Department of Electrica1, Electronics and Computer Engineering at Chiba Institute of Technology. He is a member of The Institute of E1ectrical Engineers Japan, The Institute of Electronics, Information and Communication Engineers Japan. His research fields are the electrostatic field simulation technology, the ultra-precise instrumentation on the small capacitances. 


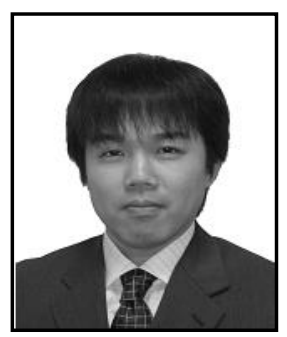

\section{Susumu Konno}

He was born 1973. He received B.E, M.E and D.E degrees in 1996, 1998 and 2002, respectively form Chiba Institute of Technology, Japan. He joined Research Institute of Electrical Communication, Tohoku University, Japan as a research associate in 2001. He was a senior assistant professor of the Graduate School of Information Sciences, Tohoku University since 2007. He has moved to Chiba Institute of Technology, Japan as an associate professor in 2008. He is an associate professor of the Department of Electrica1, Electronics and Computer Engineering at Chiba Institute of Technology. He is a member of The Institute of E1ectrical Engineers Japan, The Institute of Electronics, Information and Communication Engineers Japan, The Information Processing Society of Japan, The Institute of Electrical and Electronics Engineers, Association for the Advancement of Artificial Intelligence. His research fields are agent-based computing, knowledge engineering and symbiotic computing.

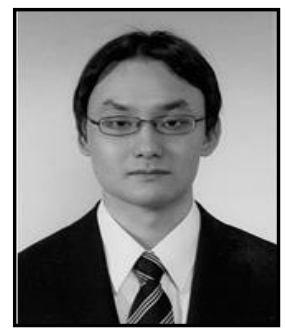

\section{Hiroaki Sugaya}

He was born 1989. He received B.E and M.E degrees in 2011 and 2013, respectively. He is with NEC Network and Sensor Systems, Ltd. Japan since April 2013.

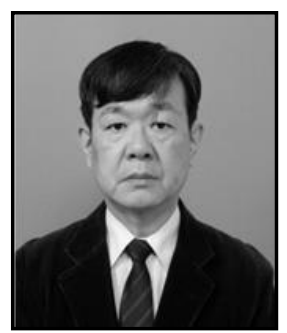

\section{Tatsuya Furukawa}

He was born 1956. He received the B .E, M.E and D.E degrees in 1979, 1981 and 1987, respectively from Kyushu University, Fukuoka, Japan. He joined Nagasaki University, Nagasaki, Japan as a research associate in 1984. He moved to Saga University, Saga, Japan as an assistant professor in 1986. He is a professor of the Department of Electrical Engineering at Saga University since 2001. $\mathrm{He}$ is a member of the Institute of Electrical Engineers Japan, the Society of Instrument and Control Engineering Japan, the Institute of Electronics, Information and Communication Engineers Japan. His research fields are the power engineering, the electric machinery, the computer science, the simulation technology, the instrumentation technology and the higher education on engineering based on the advanced computational engineering. 


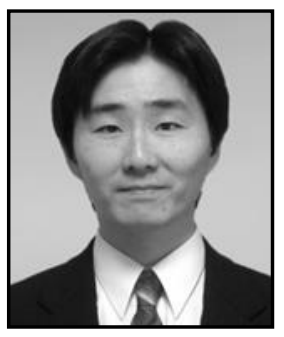

\section{Hideaki Itoh}

He was born in 1974. He received the B.E, M.E, and D.E degrees in 1997, 1999, and 2002 respectively from the University of Tokyo, Tokyo, Japan. After working as a research associate in Tokyo Institute of Technology, he has moved as an assistant professor to the Department of Electrical and Electronic Engineering at Saga University in 2009. He is a member of the Japanese Society for Artificial Intelligence, the Society of Instrument and Control Engineering Japan, the Institute of Electronics, Information and Communication Engineers Japan, Japan Society for Fuzzy Theory and Intelligent Informatics, and the Robotics Society of Japan. His research fields include artificial intelligence and soft computing.

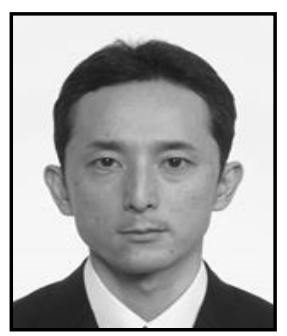

\section{Hisao Fukumoto}

He was born in 1973. He received the B.E and M.E degrees in 1997 and 1999, respectively from Saga University, Saga, Japan. He received the D.E degree from Saga University, Japan in 2002. He is a research assistant of the Department of Advanced Systems Control Engineering at Saga University since 2002. He is member of the Institute of Electronics, Information and Communication Engineers Japan, the Society of Instrument and Control Engineering Japan, the Japanese Society for Medical and Biological Engineering. His research fields are the image processing, the signal processing and the education on engineering based on the advanced computational engineering. 
International Journal of Energy, Information and Communications Vol.5, Issue 5 (2014) 